Reliability Analysis for the Facility Data

Acquisition Interface System Upgrade at TA-55

W. J. Turner, P. E.

N. G. Pope

R. E. Brown 


\section{DISCLAIMER}

This report was prepared as an account of work sponsored by an agency of the United States Government. Neither the United States Government nor any agency thereof, nor any of their employees, make any warranty, express or implied, or assumes any legal liability or responsibility for the accuracy, completeness, or usefulness of any information, apparatus, product, or process disclosed, or represents that its use would not infringe privately owned rights. Reference herein to any specific commercial product, process, or service by trade name, trademark, manufacturer, or otherwise does not necessarily constitute or imply its endorsement, recommendation, or favoring by the United States Government or any agency thereof. The views and opinions of authors expressed herein do not necessarily state or reflect those of the United States Government or any agency thereof. 


\section{DISCLAIMER}

Portions of this document may be illegible in electronic image products. Images are produced from the best available original document. 


\title{
RELIABILITY ANALYSIS FOR THE FACILITY DATA ACQUISITION INTERFACE SYSTEM UPGRADE AT TA-55
}

\author{
by
}

W. J. Turner, N. G. Pope, and R. E. Brown

\begin{abstract}
Because replacement parts for the existing facility data acquisition interface system at TA-55 have become scarce and are no longer being manufactured, reliability studies were conducted to assess various possible replacement systems. A new control system, based on Allen-Bradley Programmable Logic Controllers (PLCs), was found to have a likely reliability 10 times that of the present system, if the existing Continuous Air Monitors (CAMs) were used. Replacement of the old CAMs with new CAMs will result in even greater reliability as these are gradually phased in. The new PLC-based system would provide for hot standby processors, redundant communications paths, and redundant power supplies, and would be expandable and easily maintained, as well as much more reliable.
\end{abstract}

\section{INTRODUCTION}

The facility data acquisition interface system (FDAIS) at the Los Alamos National Laboratory Plutonium Facility is approaching the end of its expected useful life. New repair parts for most components are not available because their manufacturer has been out of business for some time. Therefore, it is necessary to consider replacing the existing system with a new system. One proposed solution is to replace the present system with a control system based on Allen-Bradley Programmable Logic Controllers (PLCs). This paper presents a reliability analysis of the PLC system and its associated hardware.

\section{EXISTING SYSTEM}

The existing system consists of 23 Field Multiplex Units (FMUs). These units are located throughout the plant. Each one has a communications connection to a Data General computer located in the TA-55 Operations Center, where all ladder logic is performed. There are approximately 2200 input/output $(\mathrm{I} / \mathrm{O})$ points connected to the existing system. 


\section{PROPOSED REPLACEMENT SYSTEM}

The replacement system consists of 19 PLCs. FMUs will be replaced by PLCs and the corresponding I/O hardware. There are four locations where two FMUs are located; one PLC can replace two FMUs in these locations. Some subsystems, such as fire alarms, may be consolidated to a single location rather than remaining distributed throughout the complex. Also, plans are underway to install a new type of Continuous Air Monitor (CAM). If these changes are made, additional PLCs will be added to the system to allow it to incorporate the CAM control functions. The goals of the design effort for a new control system were to design a system that would provide for hot standby processors, redundant communications paths, and redundant power supplies. In addition, the system must be expandable, easily maintained, and reliable. The proposed architecture is shown in Fig. 1. Replacing sensors and sensor field wiring has not been considered.

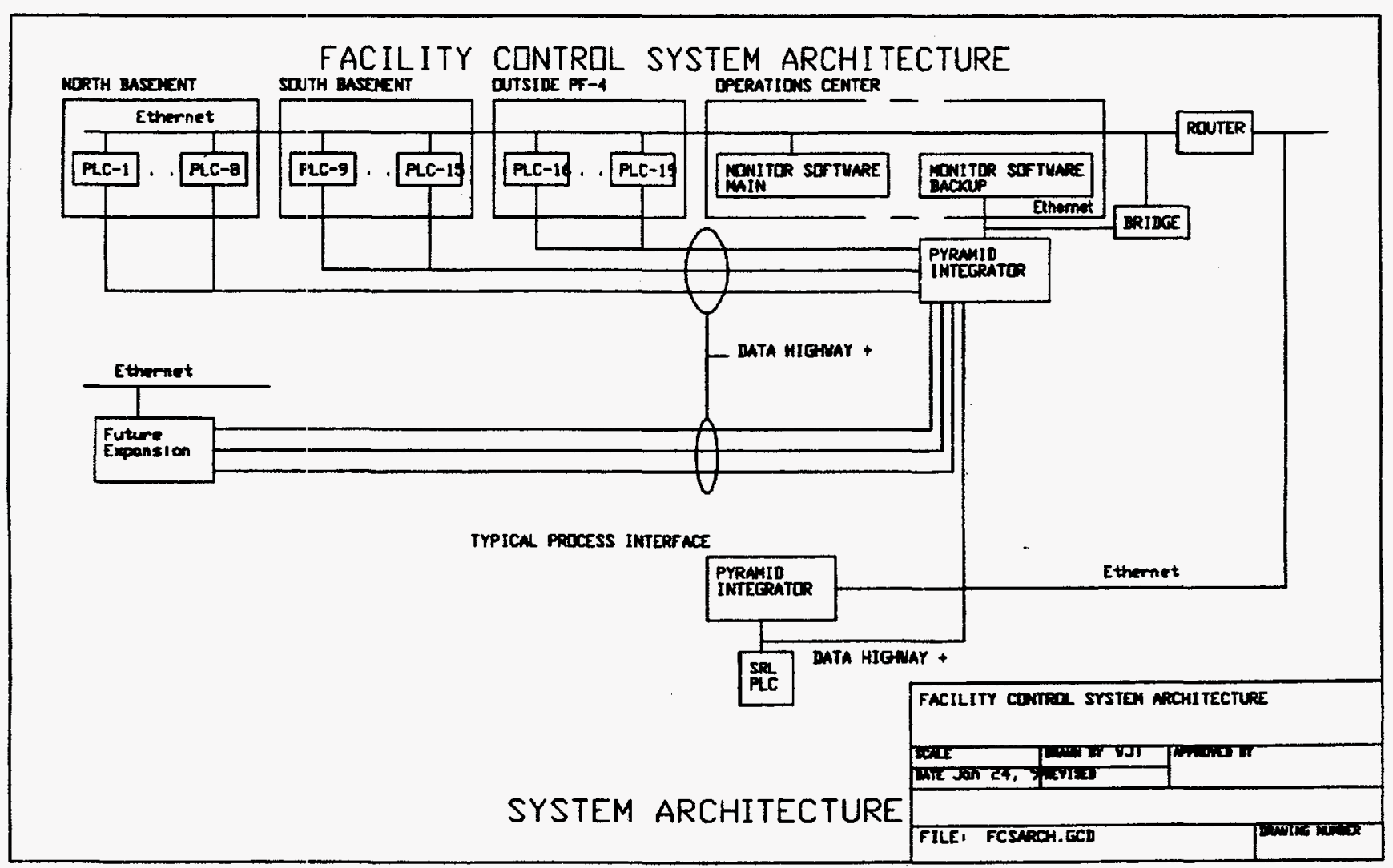

Fig. 1. Proposed facility control system architecture. 
This reliability analysis is restricted to a proposed system incorporating 19 PLC units. Several variations of the system architecture have been analyzed.

Model 1 Dual processor

Communication protocol: Ethernet and Data Highway Plus

New CAMs

Model 2 Dual processor

Communication protocol: dual Ethernet

New CAMs

Model 3 Dual processor

Communication protocol: Ethernet and Data Highway Plus

Old CAMs

Model 4 Dual processor

Communication protocol: dual Ethernet

Old CAMs

Model 5 Single processor

Communication protocol: Ethernet and Data Highway Plus

New CAMs

Model 6 Single processor

Communication protocol: dual Ethernet

New CAMs

Model $7 \quad$ Single processor

Communication protocol: Ethernet and Data Highway Plus

Old CAMs

Model 8 Single processor

Communication protocol: dual Ethernet

Old CAMs

The existing CAM has an output that is a pulse train with a rate that is proportional to the reading. This signal can be measured with a high-speed counter module. It is expected that $\sim 70$ modules will be required throughout the plant to monitor these CAMs. The new CAM, on the other hand, uses an RS485 protocol for communication and has much more data available. When the new CAMs are installed, it may be feasible to use only one communication module to obtain data from the entire CAM network. This change in module count from $\sim 70$ to 1 will provide a significant change in reliability, as shown later in this report. 


\section{DEFINITIONS}

The following definitions are used in the rest of this report. They can be found in Juran's Quality Control Handbook, Fourth Edition, J. M. Juran, Ed. (McGraw-Hill, New York, 1988).

MTBF Mean Time between Failures

MTTR Mean Time to Repair

Reliability The probability of a product performing (without failure) a specified function for a specified period of time.

Probability of Survival $\left(P_{s}\right)$, another measure of reliability, is the given by

$$
P_{s}=\exp -\frac{t}{\mu}=\exp -t \lambda
$$

where $\quad e=2.718$

$t=$ a specified period of failure-free operation

$\mu=\mathrm{MTBF}$

$\lambda=$ failure rate (reciprocal of $\mu$ ).

Availability (A) $\quad A=\frac{M T B F}{M T B F+M T T R}$.

Maintainability Probability of restoring service in the time period specified.

RAM Reliability, availability, and maintainability.

\section{THEORY}

When analyzing RAM, it is customary to start with either computed or historical data for the MTBF and MTTR. This data is required for each component of the system. The availability of each component is computed, and the system is then analyzed to determine which items are redundant. The system availability for the redundant items is computed using probability theory.

The following is an example of a RAM analysis of the system power supplies. The power supplies can be configured in sets of two, three, or four, depending on load requirements. In each case, one power supply can fail without causing failure of the system.

For the two-supply case, the analysis is straightforward. The individual availability of each supply is computed as outlined in the definition of availability. From this the probability of failure of one supply is given by

$$
P_{f}=1-A \text {. }
$$

The probability of both power supplies failing simultaneously is the calculation that is required. If the failures are independent, then the probability is the product of 
the individual probabilities, which is given by

$$
P_{\text {Both Fail }}=(1-A)^{2} \text {. }
$$

The availability of the redundant power supply is then given by

$$
A_{\text {Two-Supply System }}=1-(1-A)^{2} \text {. }
$$

The three-supply system is slightly more complicated to compute, since we must now be concerned with any two of the three failing and the remote possibility that all three would fail at the same time. Out of a total of three power supplies (if the failures are independent), there are three ways that two can fail at the same time. This is given by

$$
P_{\text {Two Fail }}=3(1-A)^{2} \text {. }
$$
by

The other possibility is that all three fail at the same time, which would be given

$$
P_{\text {Three Fail }}=(1-A)^{3} \text {. }
$$

Therefore, the probability of system failure for the three-supply system would be given by

$$
P_{\text {System Failure }}=3(1-A)^{2}+(1-A)^{3} \text {. }
$$

The availability of the three-supply system is then

$$
A_{\text {Three-Supply System }}=1-\left[3(1-A)^{2}+(1-A)^{3}\right] \text {. }
$$

Similarly, the probability of failure of the four-supply system is given by

$$
P_{\text {System Failure }}=6(1-A)^{2}+4(1-A)^{3}+(1-A)^{4},
$$

and the availability of the four-supply system is

$$
A_{\text {Four-Supply System }}=1-\left[6(1-A)^{2}+4(1-A)^{3}+(1-A)^{4}\right] \text {. }
$$

We can be thankful that our hypothetical manufacturer in this example doesn't produce a five-supply system.

We will now examine the hot-standby or redundant-PLC configuration. Figure 2 shows a single-PLC system. This consists of a

(1) chassis,

(2) PLC,

(3) Backup Communication Module, and

(4) a two-power-supply system as described above. 


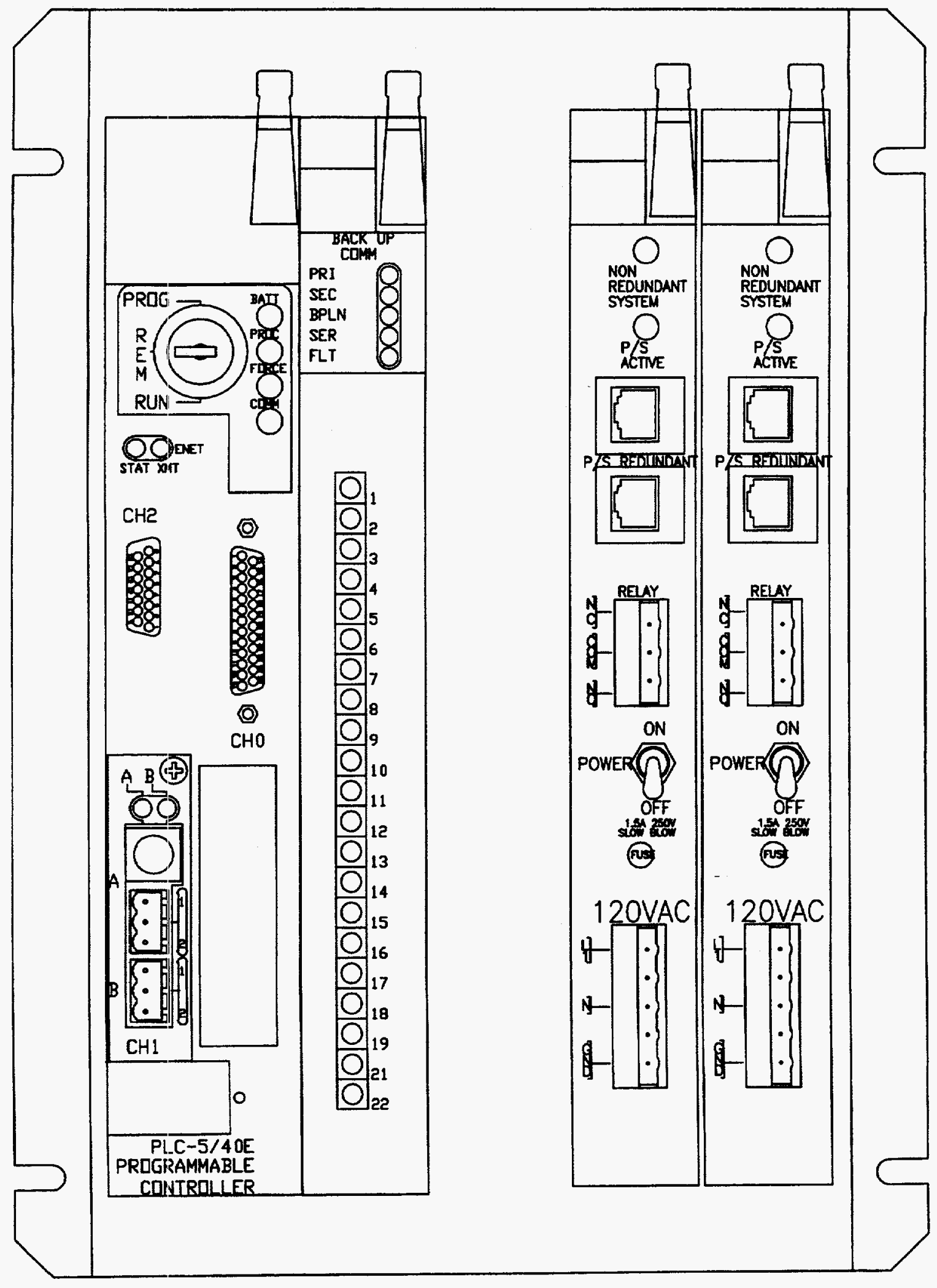

Fig. 2. A single-PLC subsystem. 
The availability for this subsystem is then

$$
\begin{aligned}
A_{\text {Single-PLC Subsystem }} & =\left(A_{\text {Chassis }}\right)\left(A_{\mathrm{PLC}}\right)\left(A_{\mathrm{BCM}}\right)\left(A_{\text {Two-Supply System }}\right) \\
& =\left(A_{\text {Chassis }}\right)\left(A_{\mathrm{PLC}}\right)\left(A_{\mathrm{BCM}}\right)\left[1-\left(1-A_{\text {Power Supply }}\right)^{2}\right] .
\end{aligned}
$$

This leads to the availability of the redundant PLC subsystem as

$$
A_{\text {Redundant PLC }}=1-\left[1-\left(1-A_{\text {Single-PLC Subsystem }}\right)^{2}\right] \text {. }
$$

\section{CALCULATIONS}

A QuickBasic program was written to make the calculations required by our reliability analysis. A copy of the program is included in Appendix A, and a sample output is shown in Appendix B.

The tables below provide a summary of our calculated results from this program. The rows correspond to the eight models, as follows:

DPNCED Model 1 (dual processor, new CAMs, Ethernet and Data Highway Plus)

DPNCEE Model 2 (dual processor, new CAMs, dual Ethernet)

DPOCED Model 3 (dual processor, old CAMs, Ethernet and Data Highway Plus)

DPOCEE Model 4 (dual processor, old CAMs, dual Ethernet)

SPNCED Model 5 (single processor, new CAMs, Ethernet and Data Highway Plus)

SPNCEE Model 6 (single processor, new CAMs, dual Ethernet)

SPOCED Model 7 (single processor, old CAMs, Ethernet and Data Highway Plus)

SPOCEE Model 8 (single processor, old CAMs, dual Ethernet).

\begin{tabular}{||c||c||}
\hline Model & MTBF (hours) \\
\hline DPNCED & 10246.89 \\
\hline DPNCEE & 10246.53 \\
\hline DPOCED & 3205.03 \\
\hline DPOCEE & 3204.99 \\
\hline SPNCED & 5715.87 \\
\hline SPNCEE & 4625.17 \\
\hline SPOCED & 2567.38 \\
\hline SPOCEE & 2321.10 \\
\hline
\end{tabular}

As indicated earlier, the MTBF for each of the models containing the new CAMs is far better than the MTBF for the models that use the old CAMs. This performance 
difference is due to the counters required for the old CAMs. The counters are complicated modules that have a low MTBF. When the CAMs are replaced, the counters will come out of the system, and the system MTBF will increase.

\begin{tabular}{|c|c|c|c|}
\hline $\begin{array}{l}\text { Probability of } \\
\text { Survival }\left(P_{s}\right)\end{array}$ & 0.37 & $\overline{0.5}$ & 0.95 \\
\hline Model & MTBF (hours) & (hours) & (hours) \\
\hline DPNCED & 10246.89 & 7102.60 & 525.60 \\
\hline DPNCEE & 10246.53 & 7102.36 & 525.58 \\
\hline DPOCED & 3205.03 & 2221.56 & 164.40 \\
\hline DPOCEE & 3204.99 & 2221.53 & 164.39 \\
\hline SPNCED & 5715.87 & 3961.94 & 293.19 \\
\hline SPNCEE & 4625.17 & 3205.92 & 237.24 \\
\hline SPOCED & 2567.38 & 1779.60 & 131.69 \\
\hline SPOCEE & 2321.10 & 1608.86 & 119.06 \\
\hline
\end{tabular}

The $P_{s}$ row indicates the probability of survival for the number of hours given. If the hypothesis is that Model 1 will survive for 525 hours without a failure, then the hypothesis can be expected to be correct $95 \%$ of the time (i.e., the model has a $P_{s}$ of 0.95 ). Since the distribution of the failures is exponential, the probability of survival to the MTBF is not 0.5 (as might be expected) but only 0.37 .

The dual processor model for the old CAMs (Model 3) shows a 0.95 probability of survival for 164 hours. This system can be contrasted with the existing system, which has a 0.95 probability of survival of only about 12 hours.

\section{CONCLUSIONS}

The old-CAM version of the new control system is at least 10 times more reliable than the existing system. This analysis has assumed that any failure would cause a system failure. This assumption is not really true because many signals only turn on status lights or have other noncritical consequences that will not take the system down.

The design that was examined uses several redundant components. The reliability results that are shown take these redundancies into account. This does not mean that individual components cannot fail or cannot fail simultaneously but means that when a redundant component fails, its function can usually be maintained without systemwide consequences. 
Appendix A

QuickBasic Program 
Reliability Analysis for the TA-55 FDAIS Upgrade 


\section{THE QUICKBASIC PROGRAM}

NOTE: The MTBF and availability numbers have been removed because the company considers those numbers to be proprietary information. The system numbers still remain.

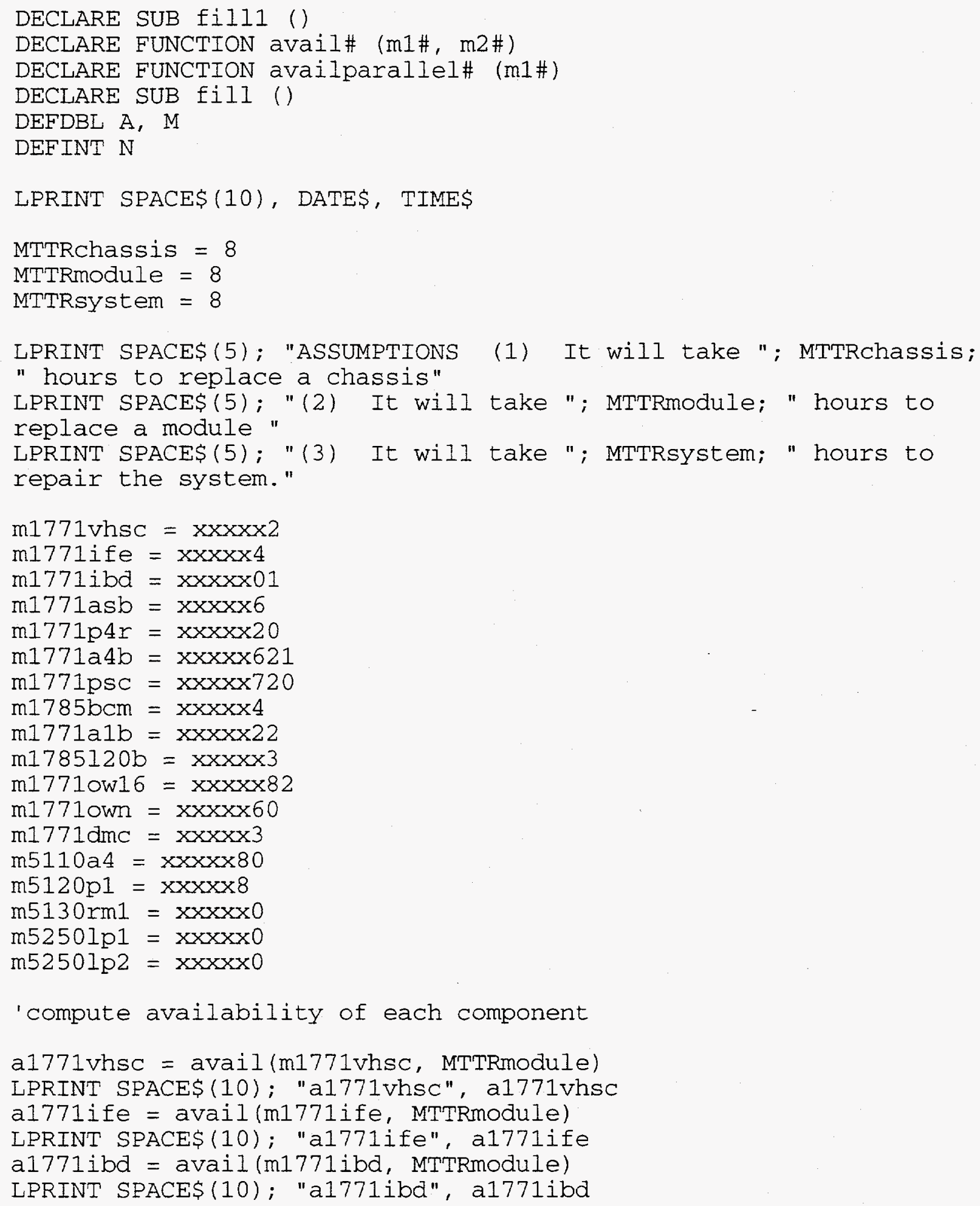


a1771asb = avail (m1771asb, MTTRmodule)

LPRINT SPACE\$(10); "a1771asb", a1771asb

a1771p4r = avail (m1771p4r, MTTRmodule)

LPRINT SPACE\$(10); "a1771p4r", a1771p4r

a1771a4b = avail (m1771a4b, MTTRchassis)

LPRINT SPACE\$(10); "a1771a4b", a1771a4b

a1771psc = avail (m1771psc, MTTRchassis)

LPRINT SPACE\$(10); "a1771psc", a1771psc a1785bcm = avail (m1785bcm, MTTRmodule)

LPRINT SPACE $(10) ;$ "a1785bcm", a1785bcm a1771alb = avail (m1771a1b, MTTRchassis) LPRINT SPACE\$ (10); "a1771a1b", a1771a1b a1785120b = avail (m1785120b, MTTRmodule) LPRINT SPACE $(10) ;$ "a1785120b", a1785120b a17710w16 = avail (m1771ow16, MTTRmodule) LPRINT SPACE $(10) ;$ "a17710w16", a17710w16 a1771own = avail (m1771own, MTTRmodule) LPRINT SPACE\$(10); "a17710wn", a17710wn al771dmc = avail (ml771dmc, MTTRmodule) LPRINT SPACE\$(10); "a1771dmc", a1771dmc

$\mathrm{n} 1=0$ 'make $\mathrm{n} 1=1$ for single processor, make $\mathrm{n} 1=0$ for dual processor

$\mathrm{n} 2=1$ 'make $\mathrm{n} 2=1$ for old CAM, make $\mathrm{n} 2=0$ for new CAM

$\mathrm{n} 3=0$ 'make $\mathrm{n} 3=1$ for dual ethernet, make $\mathrm{n} 3=0$ for ethernet $\mathrm{DH}+$

FOR $\mathrm{n} 1=0$ TO 1

FOR $\mathrm{n} 2=0$ TO 1

FOR $n 3=0$ TO 1

$$
\begin{aligned}
& \text { IF n3 THEN } \\
& \text { nee }=1 \\
& \text { ned }=0 \\
& \text { ELSE } \\
& \text { nee }=0 \\
& \text { ned }=1 \\
& \text { END IF }
\end{aligned}
$$

$$
\begin{aligned}
& \text { IF n1 THEN } \\
& \text { nSp }=1 \\
& \text { ndp }=0 \\
& \text { ELSE } \\
& \text { nSp }=0 \\
& \text { ndp }=1 \\
& \text { END IF } \\
& \text { IF n2 THEN } \\
& \text { nOC }=1 \\
& \text { nnC }=0 \\
& \text { ELSE } \\
& \text { nOC }=0 \\
& \text { nnC }=1 \\
& \text { END IF }
\end{aligned}
$$




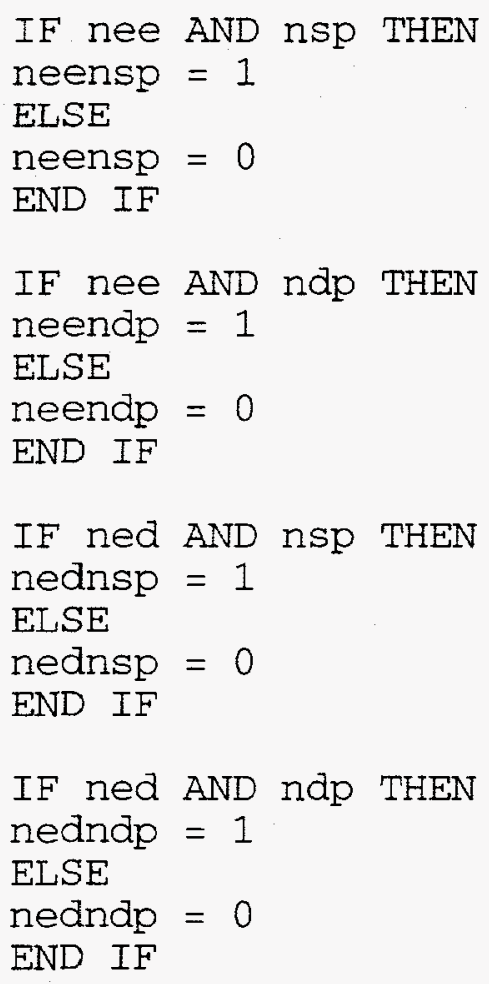

IF nsp = 1 THEN LPRINT SPACE\$(5); "This calculation uses a single processor model"

IF ndp = 1 THEN IPRINT SPACE\$(5); "This calculation uses a dual processor model"

IF nOC $=1$ THEN LPRINT SPACE\$ (5); "This calculation uses the old CAM model"

IF nnC $=1$ THEN LPRINT SPACE\$(5); "This calculation uses the new CAM model"

IF nee $=1$ THEN LPRINT SPACE\$ (5); "This calculation uses dual ethernet model"

IF ned = 1 THEN LPRINT SPACE\$(5); "This calculation uses ethernet and Data Highway+ model"

'calculate redundant power supply availability

ap1771p4r = availparallel (a1771p4r)

LPRINT SPACE\$(10); "ap1771p4r", ap1771p4r

'calculate single path availibility for dual processor configuration

availsp $=$ ap1771p4r * a1785bcm * a1785120b * a1771a1b

LPRINT SPACE\$(10); "availsp", availsp 
'calculate parallel path availability for dual processor configuration

availdp = avajlparallel (availsp)

LPRINT SPACE\$(10); "availdp", availdp

calculate powersupply availibility for remote i/o (four supplies)

DIM SHARED atotaledplc(20), modules $(20,3), \operatorname{nCAM}(20,4)$

apsrio(4) = a1771psc * availparallel(a1771p4r ^ 2)

LPRINT SPACE\$(10); "apsrio(4)", apsrio(4)

'calculate powersupply availability for remote i/o (two supplies)

apsrio(2) = a1771psc * availparallel (a1771p4r)

LPRINT SPACE\$(10); "apsrio(2)", apsrio(2)

availspee $=$ apsrio $(2)$ * a1771alb * a1785120b * a1771dmc *

a1785 bcm

availdpee = availparallel (availspee)

availsystem $=1$

CALL fill1

CALL fill

FOR $i=1$ TO 1.9 STEP 1

atotaledplc $(i)=$ availdpee $\wedge$ neendp * availspee $\wedge$ neensp * a1771a4b * availdp ^ nedndp * availsp ^ nednsp * a1771asb * al771ife ^ modules(i, 1) * al771ibd ^ modules(i., 2) * al771own ^ modules $(i, 3)$ *apsrio $(\operatorname{nCAM}(i$, noc) $)$ *a1771vhsc $\wedge$ (noc * nCAM(i

(2))

LPRINT SPACE\$(10); "atotaledplc"; i atotaledplc(i)

availsystem = availsystem * atotaledplc(i)

NEXT

LPRINT SPACE\$(10); "availsystem" ; availsystem

mtbfsystem = MTTRsystem * availsystem / (1 - availsystem $)$

LPRINT SPACE\$(10); "mtbfsystem", mtbfsystem

LPRINT CHR\$ (12)

NEXT

NEXT

NEXT

DEFSNG $N$

FUNCTION avail (m1, m2) STATIC

avail $=\mathrm{m} 1 /(\mathrm{m} 1+\mathrm{m} 2)$

END FUNCTION 
FUNCTION availparallel (m1)

availparallel $=1-(1-\mathrm{m} 1) \wedge 2$

END FUNCTION

SUB fill

'modules $(n, i)=v a l u e ~ n$ is PLC \#,

' for $i=1$ value is number of analog modules in rio of PLC \#n

- for $i=2$ value is number of discrete input modules in rio of PLC \#n

' for $i=3$ value is number of discrete output modules in rio of PLC\#n

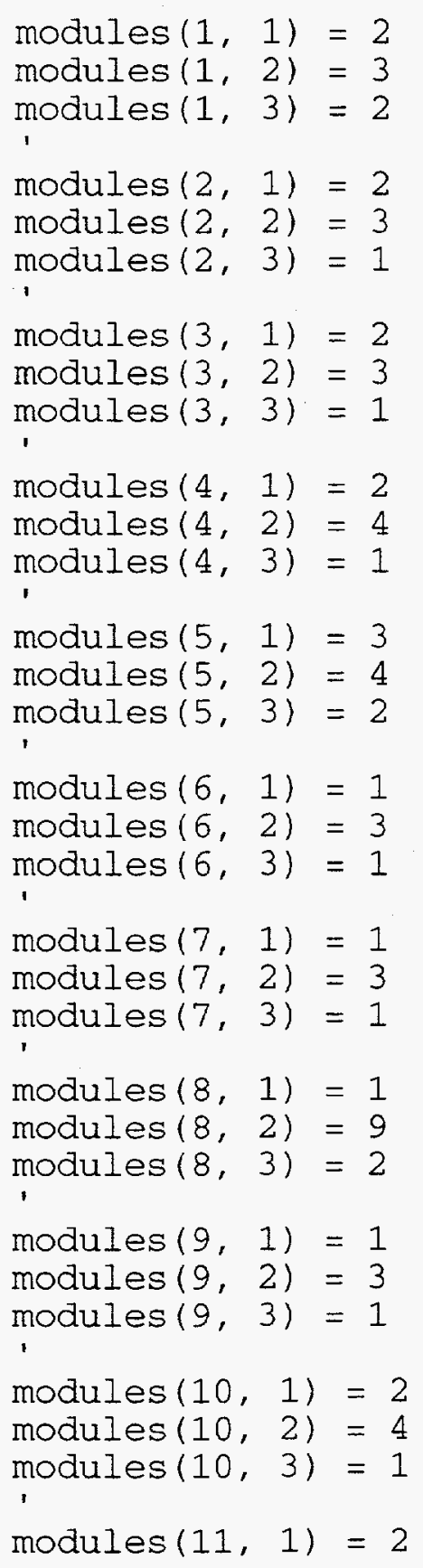




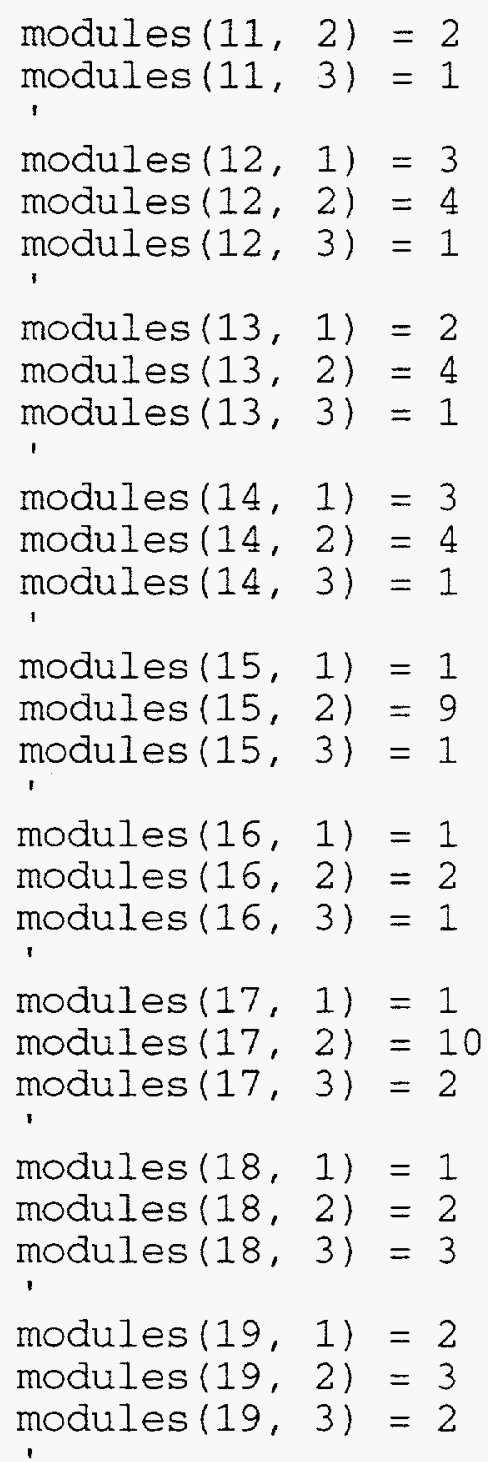




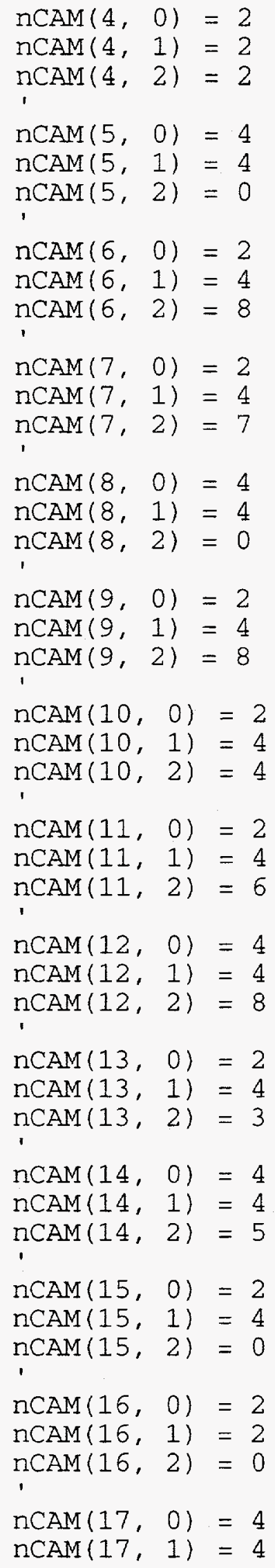


Reliability Analysis for the TA-55 FDAIS Upgrade

$\operatorname{nCAM}(17,2)=0$
$\operatorname{nCAM}(18,0)=4$
$\operatorname{nCAM}(18,1)=4$
$\operatorname{nCAM}(18,2)=0$
$\operatorname{nCAM}(19,0)=4$
$\operatorname{nCAM}(19,1)=4$
$\operatorname{nCAM}(19,2)=0$
1

END SUB 
Appendix B

Sample Output 
Reliability Analysis for the TA-55 FDAIS Upgrade 
SAMPLE OUTPUT chassis

ASSUMPTIONS (1) It will take 8 hours to replace a

(2) It will take 8 hours to replace a module

(3) It will take 8 hours to repair the system. a.1771vhsc a1771ife a1771ibd a1771asb a1771p4r a.1771a $4 \mathrm{~b}$ a.1771psc a1785bcm a.1771a1b a1785120b a.17710w16 a17710wn a.1771dmc . $x \times x \times x 48158408361$ . $\mathrm{xxx \times x} 0674599474$ $. \times \times x \times x 7847268547$ . $x \times x \times x 1128794381$ $. \times \times \times x \times 39275618858$ . $x \times x \times x 94585312429$ . $\times x \times x \times 94372034414$ . $x \times x \times x 9556708379$

$. \times x \times x \times 86933933066$

$. \times x \times x \times 92116539468$

$. \times \times \times x \times 58480166494$

$. \times \times \times x \times 56516256397$

. $x \times x \times x 32186960508$

This calculation uses a dual processor model

This calculation uses the new CAM model

This calculation uses ethernet and Data Highway+ model ap1771p4r availsp availdp apsrio (4) apsrio(2) atotaledplc 1 atotaledplc 2 atotaledplc 3 atotaledplc 4 atotaledplc 5 atotaledplc 6 atotaledplc 7 atotaledplc 8 atotaledplc 9 atotaledplc 10 atotaledplc 11 atotaledplc 12 atotaledplc 13 atotaledplc 14 atotaledplc 15 atotaledplc 16 atotaledplc 17 atotaledplc 18 atotaledplc 19 availsystem .999219884446688 . $x \times x \times x 99999631255$ $. \times x \times x \times 7461976665$

$. \times \times \times x \times 9998941277$

$. \times \times \times x \times 94370559444$

$. \times x \times x \times 94371665669$

.999956218384679

.9999605666981861

.9999605666981861

.9999584140516224

.9999447407753053

.9999698918179413

.9999698918179413

.9999526275897312

.9999698918179413

.9999584140516224

.9999627193493839

.9999490889282852

.9999584140516224

.9999490889282852

.9999569758876239

.9999720444892136

.9999504749602584

.9999633478919044

.999956218384679

mtbfsystem

10246.89104278906 
This calculation uses a dual processor model This calculation uses the new CAM model

This calculation uses dual ethernet model ap1771p4r availisp ava:ildp apsicio(4) apsicio (2) atotaledplc $1 \quad .9999562169552955$ atotaledplc $2 \quad .9999605652687965$ atotaledplc $3 \quad .9999605652687965$ atotaledplc $4 \quad .9999584126222359$ atotaledplc $5 \quad .9999447393459383$ atotaledplc $6 \quad .9999698903885383$ atotaledplc $7 \quad .9999698903885383$ atotaledplc $8 \quad .999952626160353$ atotaledplc $9 \quad .9999698903885383$ atotaledplc $10 \quad .9999584126222359$ atotaledplc $11 \quad .9999627179199911$ atotaledplc $12 \quad .9999490874989119$ atotaledplc $13 \quad .9999584126222359$ atotaledplc $14 \quad .9999490874989119$ atotaledplc $15 \quad .9999569744582394$ atotaledplc $16 \quad .9999720430598076$ atotaledplc $17 \quad .9999504735308831$ atotaledplc $18 \quad .9999633464625108$ atotaledplc $19 \quad .9999562169552955$ ava:L Isystem . 9992198573084018 mtbisystem

10246.53431296156 
This calculation uses a dual processor model

This calculation uses the old CAM model

This calculation uses ethernet and Data Highway+ model ap1771p4r availsp availdp apsrio (4) apsrio(2) . xxxxx99999631255 .$\times \times \times \times \times 7461976665$ . $\times x \times x \times 9998941277$ $. \times x \times x \times 94370559444$ atotaledplc 1 atotaledplc 2 atotaledplc 3 atotaledplc 4 atotaledplc 5 atotaledplc 6 atotaledplc 7 atotaledplc 8 atotaledplc 9 atotaledplc 10 atotaledplc 11 atotaledplc 12 atotaledplc 13 atotaledplc 14 atotaledplc 15 atotaledplc 16 atotaledplc 17 atotaledplc 18 atotaledplc 19 .$\times \times \times \times \times 94371665669$

.9997799503066913 .9998598377285391 .9998094771041023 .9999080484621246 .9999447407753053 .9997684422573708 9997936212190586 .9999526275897312 9997684422573708 .9998576852988179 .999811629430044 .9997476436691685 .9998828665080732 .9998231808851646 .999956975777006 .9999720444892136 .9999504749602584 .9999633478919044 availsystem .9975101394724409 mtbfsystem

3205.031377240559 


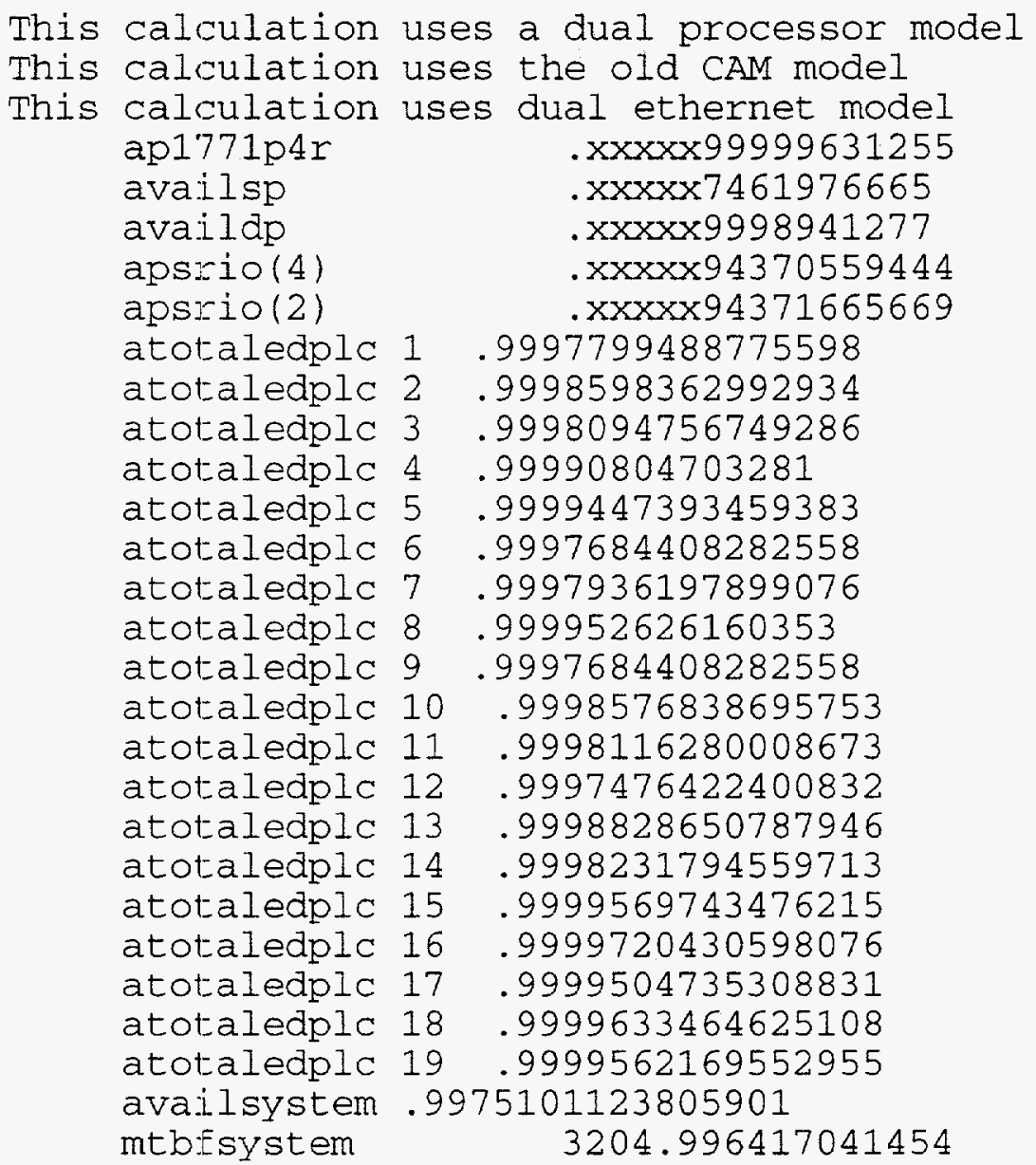


This calculation uses a single processor model

This calculation uses the new CAM model

This calculation uses ethernet and Data Highway+ model ap1771p4r

availsp

availdp

apsrio(4)

apsrio(2)

. $x \times x \times x \times 99999631255$

$. \times x \times x \times 7461976665$

$. \times \times \times x \times 9998941277$

$. \times x \times x \times 94370559444$

atotaledplc $1 \quad .9999236828445534$

atotaledplc $2 \quad .9999280310165796$

atotaledplc $3 \quad .9999280310165796$

atotaledplc $4 \quad .9999258784400565$

atotaledplc $5 \quad .9999122056086263$

atotaledplc $6 \quad .9999373558329236$

atotaledplc $7 \quad .9999373558329236$

atotaledplc $8 \quad .9999200921664392$

atotaledplc $9 \quad .9999373558329236$

atotaledplc $10 \quad .9999258784400565$

atotaledplc $11 \quad .9999301835977367$

atotaledplc $12 \quad .9999165536201304$

atotaledplc $13 \quad .9999258784400565$

atotaledplc $14 \quad .9999165536201304$

atotaleaplc $15 \quad .9999244403228514$

atotaledplc $16 \quad .9999395084341546$

atotaledplc $17 \quad .9999179396070063$

atotaledplc $18 \quad .9999308121198063$

atotaledplc $19 \quad .9999236828445534$

availsystem .9986023452429307

mtbfsystem

5715.874196782925 
This calculation uses a single processor model This calculation uses the new CAM model This calculation uses dual ethernet model ap1771p4r availsp availdp apsrio(4) apsrio(2) .xxxxx99999631255 $. x \times x \times x 7461976665$ . $\operatorname{xxxxx} 9998941277$ $. \times \times x \times x \times 4370559444$ atotaledplic $1 \quad .9999063400771416$ atotaledplc $2 \quad .9999106881737528$ atotaledplc $3 \quad .9999106881737528$ atotaledplc $4 \quad .9999085356345643$ atotaledplc $5 \quad .9998948630402769$ atotaledplc $6 \quad .9999200128283665$ atotaledplc $7 \quad .9999200128283665$ atotaledplc $8 \quad .9999027494613046$ atotaledplc $9 \quad .9999200128283665$ atotaledplc $10 \quad .9999085356345643$ atotaledplc $11 \quad .9999128407175754$ atotaledplc $12 \quad .9998992109763687$ atotaledplc $13 \quad .9999085356345643$ atotaledplc $14 \quad .9998992109763687$ atotaledplc $15 \quad .999907097542302$ atotaledplc $16 \quad .9999221653922624$ $\begin{array}{lll}\text { atotaledplc } & 17 & .9999005969392059\end{array}$ atotaledplc $18 \quad .9999134692287439$ atotaledplc $19 \quad .9999063400771416$ availsystem .9982733194555519 mtbfsystem 4625.167394931695 
This calculation uses a single processor model

This calculation uses the old CAM model

This calculation uses ethernet and Data Highway+ model ap1771p4r

availsp

availdp

apsrio(4)

. $\times \times \times x \times 99999631255$

.$\times \times \times \times \times 7461976665$

.$\times \times \times \times x 9998941277$

apsrio(2)

. $x \times x \times x 94370559444$

atotaledplc 1

atotaledplc 2

atotaledplc 3

atotaledplc

atotaledplc 5

atotaledplc 6

atotaledplc 7

atotaledplc 8

atotaledplc 9

atotaledplc 10

. $\mathrm{x} \times x \times x 94371665669$

atotaledplc 11

atotaledplc 12

atotaledplc 13

atotaledplc 14

atotaledplc 15

atotaledplc 16

atotaledplc 17

.9997474205017939

.9998273053243475

.9997769463384926

.999875514489302

.9999122056086263

.9997359128269104

.9997610909693512

.9999200921664392

.9997359128269104

.9998251529646598

.9997790985944042

.999715114915431

.9998503333545951

.9997906496736755

.9999244402122371

.9999395084341546

atotaledplc

atotaledplc $19 \quad .9999236828445534$

availsystem .9968936569275494

mtbfsystem

2567.375550418117 


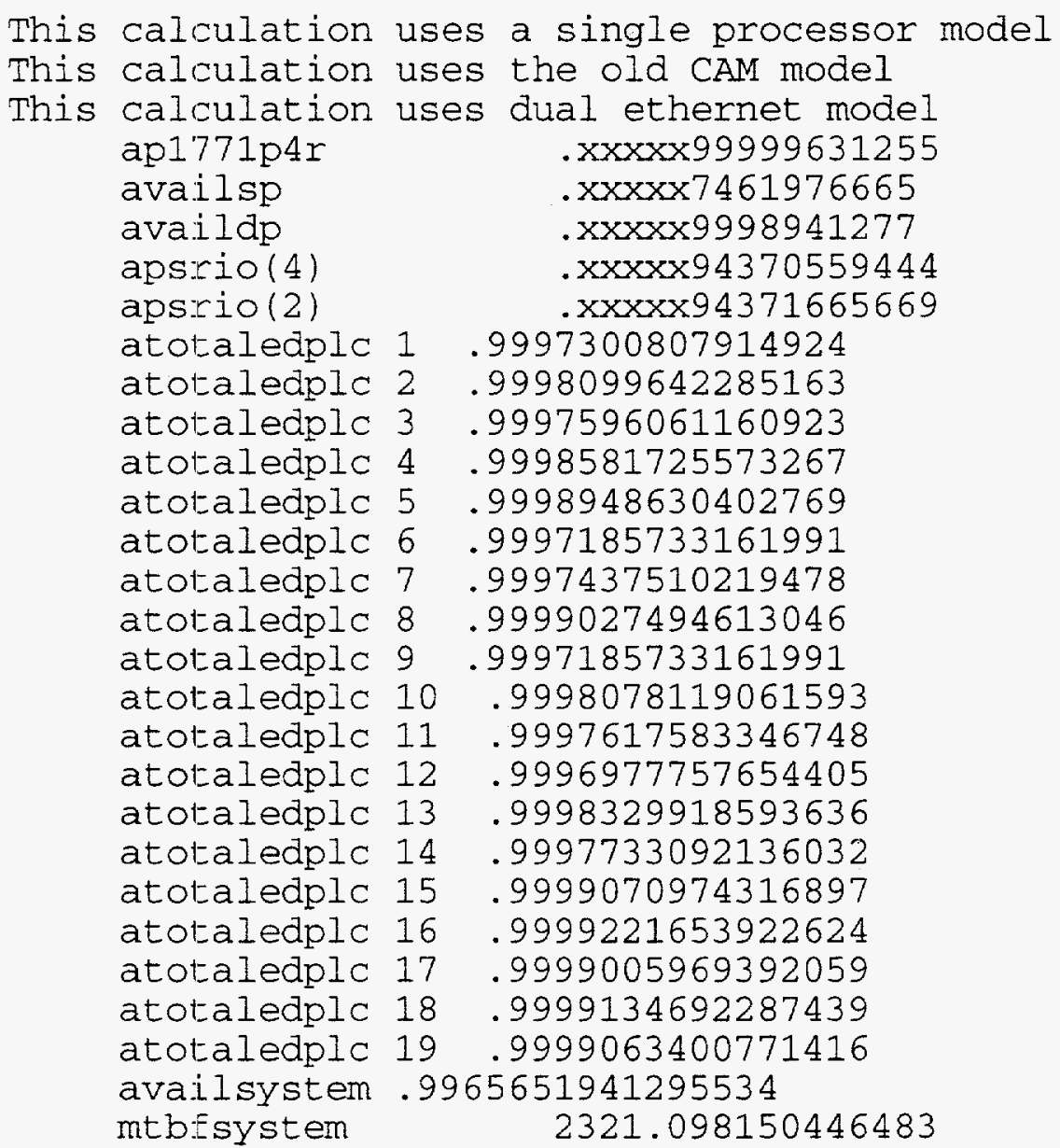

